\title{
Philosophiques
}

\section{Arturo Sangalli, Éloge du flou. Aux frontières des mathématiques et de l'intelligence artificielle, Montréal, Les Presses de l'Université de Montréal, 2001, 206 pages.}

\section{Dominic Forest}

Volume 31, numéro 2, automne 2004

URI : https://id.erudit.org/iderudit/009823ar

DOI : https://doi.org/10.7202/009823ar

Aller au sommaire du numéro

Éditeur(s)

Société de philosophie du Québec

ISSN

0316-2923 (imprimé)

1492-1391 (numérique)

Découvrir la revue

Citer ce compte rendu

Forest, D. (2004). Compte rendu de [Arturo Sangalli, Éloge du flou. Aux frontières des mathématiques et de l'intelligence artificielle, Montréal, Les Presses de l’Université de Montréal, 2001, 206 pages.] Philosophiques, 31(2), 444-446. https://doi.org/10.7202/009823ar d'utilisation que vous pouvez consulter en ligne.

https://apropos.erudit.org/fr/usagers/politique-dutilisation/ 


\section{Arturo Sangalli, Éloge du flou. Aux frontières des mathématiques et de l'intelligence artificielle, Montréal, Les Presses de l'Université de Montréal, 2001, 206 pages.}

Cet ouvrage, publié initialement en 1998 chez Princeton University Press sous le titre The importance of being fuzzy and other insights from the border between math and computers, est une introduction au domaine du calcul souple (soft computing), lequel fait l'objet, depuis plusieurs années déjà, d'intensives recherches, tant en sciences cognitives et en mathématiques que dans les domaines, plus appliqués, de l'informatique et de l'intelligence artificielle.

Il ne vise pas à introduire le lecteur à l'ensemble de la problématique du calcul souple, mais plutôt à présenter les fondements théoriques, ainsi que les applications de trois types spécifiques de méthodes "souples" permettant de résoudre des problèmes qui échappent aux méthodes, plus classiques, dites "exactes ». Les méthodes auxquelles s'attarde l'auteur sont : 1) la logique floue, 2) les réseaux de neurones artificiels (RNA), 3) les algorithmes génétiques (AG). La particularité de ces approches «souples » ou plus flexibles réside, comme le souligne l'auteur (p. 12), dans leur possibilité de traiter des données ambiguës ou incertaines, le parallélisme massif, le hasard et les solutions approximatives.

Le livre de Sangalli est divisé en trois parties, chacune étant composée de deux chapitres. Dans la première, l'auteur présente les principes généraux de la logique floue et de la théorie des ensembles flous. Le premier chapitre permet au lecteur, tout d'abord, de bien situer la problématique à l'égard de la théorie des ensembles, de la logique classique et de l'algèbre booléenne. Par la suite, l'auteur présente de manière claire et succincte la théorie des ensembles flous ainsi que certaines des opérations élémentaires dans cette théorie. Sans entrer dans des considérations théoriques et techniques qui seraient certainement inappropriées dans un tel ouvrage de type introductif, Sangalli consacre l'essentiel du second chapitre à la présentation d'applications concrètes qui intègrent plusieurs des principes abordés dans le chapitre précédent. La présentation de ces exemples d'applications est menée de manière détaillée et efficace, permettant ainsi de bien comprendre les principes plus abstraits de la logique floue. Ce chapitre n'est cependant pas entièrement dénué de contenu théorique. En effet, on y retrouve une très bonne présentation des principes et de la formalisation mathématique de l'inférence floue.

La deuxième partie de l'ouvrage est consacrée à la présentation des fondements mathématiques et des limites du calcul traditionnel. Selon Sangalli, il est nécessaire d'aborder ces questions afin de bien saisir les enjeux et toute la pertinence des méthodes et techniques présentées dans les chapitres 1 (la logique floue) et 3 (les réseaux de neurones artificiels et les algorithmes génétiques). Dans le troisième chapitre, l'auteur se propose donc d'effectuer un tour d'horizon des principaux concepts, enjeux et limites de l'informatique classique. On y retrouve entre autres une bonne présentation des machines de Turing ainsi qu'une réflexion sur la calculabilité des fonctions. Dans le chapitre suivant sont abordés plusieurs principes relevant des fondements des mathématiques, dont une description du théorème d'incomplétude de Gödel.

Cette deuxième partie de l'ouvrage aborde des enjeux qui permettent effectivement de bien contextualiser les méthodes de calculs souples présentées dans les autres sections. De plus, et c'est là une des grandes forces de l'ouvrage de Sangalli, les concepts traités, malgré leur complexité, sont présentés avec une aisance qui en facilite certainement 
la compréhension. Cependant, lorsque l'on compare le contenu de cette partie avec celui de la première et de la troisième partie, il est un peu surprenant de retrouver de telles considérations dans le cadre de cet ouvrage. En effet, la deuxième partie se distingue radicalement des deux autres. Les exemples y sont manifestement moins nombreux, et la complexité des concepts abordés dépasse largement celle des concepts abordés ailleurs dans l'ouvrage. Que le lecteur néophyte cherchant d'abord et avant tout une vue d'ensemble de la logique floue, des réseaux de neurones artificiels et des algorithmes génétiques en soit averti. Cette partie de l'ouvrage, bien que pertinente et bien menée, lui semblera probablement plus aride et plus difficilement accessible.

Comme il a été mentionné précédemment, la dernière partie de l'ouvrage vise à introduire le lecteur aux réseaux de neurones artificiels (RNA) et aux algorithmes génétiques (AG). Dans le chapitre 5, l'auteur présente clairement - et sans s'attarder à des détails techniques souvent caractéristiques des ouvrages informatiques sur les RNA quelques principes généraux des RNA afin d'exposer par la suite le concept d'algorithme d'apprentissage. Encore une fois, comme dans la première section, la majorité des concepts abordés sont accompagnés de plusieurs exemples fort pertinents (reconnaissance de formes, détection de programmes informatiques contenant des virus, problème du commis voyageur) permettant au lecteur de bien les saisir. Ce chapitre constitue manifestement une très bonne introduction non technique aux principes et aux applications possibles des RNA. Nous regrettons cependant que l'auteur n'ait pas développé davantage sa présentation des RNA en abordant les principes et distinctions entre les différentes architectures neuronales. Cependant, cette lacune est largement compensée par la qualité d'exposition des concepts abordés.

Le dernier chapitre est consacré aux algorithmes génétiques. On y retrouve le style et l'aisance caractéristiques du chapitre précédent. Sans entrer dans les détails techniques de la génétique, Sangalli présente les parallèles entre l'évolution génétique des organismes vivants et les solutions informatiques inspirées de la génétique. Encore une fois, les principaux concepts du domaine sont abordés et finement expliqués. Les processus de croisement et de mutation sont particulièrement bien présentés et sont soutenus par des exemples pertinents. La présentation de l'algorithme ne fait pas exception à cet égard. En ayant recours à des exemples comme ceux du dilemme du prisonnier ou du commis voyageur, Sangalli démontre clairement comment les différentes techniques présentées peuvent constituer des solutions souvent complémentaires à un même problème.

Notre appréciation générale de l'ouvrage de Sangalli ne peut être que fort positive. Les sections 1 et 3 de l'ouvrage (portant respectivement sur la logique floue d'une part et les réseaux de neurones et les algorithmes génétiques d'autre part) constituent d'excellentes introductions générales aux problématiques abordées. La deuxième partie de l'ouvrage est tout aussi pertinente eu égard aux objectifs de l'auteur, mais elle semblera malheureusement peut-être un peu moins accessible pour le lecteur ne possédant qu'une connaissance limitée des enjeux mathématiques dans leur application à l'intelligence artificielle. Par ailleurs, les nombreux enjeux cognitifs soulevés par les approches abordées ne sont qu'indirectement introduits par l'auteur. Il s'agit là manifestement d'un geste volontaire avec lequel nous ne pouvons qu'être en accord. En procédant ainsi, Sangalli atteint son objectif initial — celui d'initier le lecteur aux domaines de la logique floue, des RNA et des AG - tout en l'invitant à consulter des ressources plus ciblées dans le domaine des sciences cognitives. Nous recommandons donc vivement la lecture de cet ouvrage à toute personne travaillant dans les domaines 
des sciences cognitives et de l'intelligence artificielle et voulant s'initier aux principes généraux de la logique floue et des méthodes souples, qui permettent de résoudre des problèmes plus difficilement traitables par les méthodes classiques.

DOMINIC FOREST

Université du Québec à Montréal

\section{Philip Knee, La parole incertaine: Montaigne en dialogue, Québec, Presses de l'Université Laval, 2003.}

Comment rendre compte de la pensée de Montaigne sans la réduire à un système philosophique dans lequel elle perd son originalité et même son sens? Dans un court avant-propos, Philip Knee indique que la réflexion qu'il a menée n'est ni une introduction à l'œuvre, ni un ouvrage d'érudition, encore moins une étude fouillée du scepticisme de Montaigne, mais une recherche sur l'art du jugement qui tente à la fois de prendre au sérieux les positions morales et politiques de l'auteur des Essais et le caractère ouvert de l'écriture montaigniste, afin d'éviter, grâce à ses précautions, l'esprit de système. Le livre de M. Knee parvient bien en effet à garder ses distances face à cette menace qui guette toute étude de Montaigne : fournir des explications synthétiques et réductrices qui ferment sur lui-même le projet philosophique des Essais. Prenant pour fil conducteur l'incertitude de la pensée, cette étude permet de considérer la conversion intellectuelle qui s'opère dans les Essais, sans tirer des conclusions définitives. Cette approche permet un dévoilement clair et souple à la fois des problèmes et des enjeux moraux et politiques rencontrés dans l'ouvrage.

Dans une première section dédiée à Montaigne seul, l'identification de la pensée des Essais grâce à des verbes pronominaux réfléchis, "se dédoubler ", " s'essayer ", "se raconter ", permet de donner à la réflexion une certaine unité sans imposer une hypothèse extérieure au projet montaigniste. Dans chaque cas, M. Knee établit le lien entre l'action et l'incertitude. Ainsi l'acte réflexif du dédoublement est renvoyé à celui de l'interrogation sur les modes d'appréhension de la réalité comme sur la remise en question de nos possibilités de savoir; l'écriture de l'essai permet à Montaigne d'éviter le dogmatisme d'une certaine philosophie comme elle permet d'assumer l'ignorance et l'incomplétude de l'homme; l'objectif de se raconter, dirigé par la nature de l'essai, se réalise alors tout en réfléchissant les difficultés qu'il ne peut éliminer, celui du caractère rhétorique et artistique du portrait naturel notamment. La première partie de l'ouvrage de M. Knee, qui donne les bases afin d'interpréter les dialogues qui suivent avec Machiavel, Pascal, Rousseau et Diderot remplit ainsi son objectif : il montre en quoi la forme de l'essai est adaptée aussi bien à la nature politique et morale du propos de Montaigne qu'à son but strictement zététique. Cette section de l'ouvrage n'est pas sans présenter quelques difficultés toutefois. La présentation très concise, soulève des pistes d'interprétations intéressantes mais sans toujours fournir les outils argumentatifs attendus. Sans entrer dans une analyse du scepticisme de Montaigne, mais s'appuyant vraisemblablement sur le postulat de ce scepticisme dans toute l'œuvre, M. Knee fait l'économie d'une définition et d'une justification de l'incertitude. Si l'on mentionne souvent un lien entre incertitude et inconstance, on ne dit rien de l'irrésolution qui apparaît pourtant comme une manière bien singulière de présenter le doute philosophique dans les Essais. On regrette que le chapitre sur «l'exercice du jugement dans les Essais» ne soit pas plus étendu. 